\title{
Endometriosis with an aberrant immunophenotype: Challenging differential diagnosis of glandular lesions in the pelvic lymph nodes
}

\author{
Dita VIckovaa , Jiri Lenz $z^{\mathrm{b}, c, d}$, Radek Chvatal ${ }^{\mathrm{a}}$, Jan Tihon ${ }^{\mathrm{e}}$, Miroslav Kavka ${ }^{\mathrm{e}}$, Lucie Uncapher ${ }^{\mathrm{f}}$
}

\begin{abstract}
Case report. We describe an unusual case of pelvic lymph node endometriosis with an aberrant immunophenotype mimicking metastasis of adenocarcinoma. A 37-year-old patient with a history of invasive cervical adenocarcinoma stage pT1a2 is presented. Due to insufficient loop electrosurgical excision procedure (LEEP) conization, total laparoscopic hysterectomy with pelvic lymphadenectomy was indicated. Intraoperatively, the diagnosis of deep infiltrating endometriosis of parametrial ligament and vesicouterine pouch, endometrioma of the left ovary and Allen Master's syndrome was suspected; the patient had no history or clinical symptoms of endometriosis. A PubMed search of similar cases was followed by a comparison to this case and discussion of the differential diagnosis of glandular lesions in the pelvic lymph nodes is reported.

Results. Histological investigation showed no residual neoplasia; the diagnosis of endometriosis was confirmed. An interesting microscopic finding was represented by a solitary glandular lesion in one pelvic lymph node. Using immunohistochemistry, it was demonstrated that there was a complete loss of oestrogen and progesterone receptor expression (unlike parametrial ligament endometriosis). The diagnosis of endometriosis was based on the presence of endometrial stroma; malignancy was excluded by bland cytomorphologic features and results of immunohistochemical examination.

Conclusions. This type of aberrant of the endometriotic gland immunophenotype has never been presented in the scientific literature before. This finding plays a significant role from the pathology standpoint and, perhaps more importantly, from the clinical standpoint. An asymptomatic patient with a correct diagnosis of lymph node endometriosis did not undergo excessive treatment for false positive diagnosis of metastatic cervical adenocarcinoma.
\end{abstract}

Key words: endometriosis, aberrant immunophenotype, oestrogen and progesterone receptors, adenocarcinoma, differential diagnosis.

Received: February 2, 2017; Accepted: July 11, 2017; Available online: August 24, 2017

https://doi.org/10.5507/bp.2017.032

${ }^{a}$ Department of Obstetrics and Gynaecology, Znojmo Hospital, Czech Republic

${ }^{b}$ Department of Pathology, Znojmo Hospital, Czech Republic

'Cytohisto s.r.o., Breclav, Czech Republic

${ }^{d}$ Department of Anatomy, Histology, and Embryology, Faculty of Veterinary Medicine, University of Veterinary and Pharmaceutical Sciences, Brno, Czech Republic

eDepartment of Surgery, Znojmo Hospital, Czech Republic

${ }^{f}$ Department of Internal Medicine, Poudre Valley Hospital, Fort Collins, CO, USA

Corresponding author: Jiri Lenz, e-mail:jiri.lenz@gmail.com

\section{INTRODUCTION}

Endometriosis represents a common, chronic, estrogen-dependent gynaecologic disorder and is defined as the presence of endometriotic glandular and stromal tissue outside the uterine corpus ${ }^{1-3}$. It is found in approximately $5-10 \%$ of premenopausal women ${ }^{3,4}$.

The typical symptoms of endometriosis include chronic pelvic pain of varying intensity (50-90\% of women), infertility (20-50\% of women), dysmenorrhea ( $50 \%$ of women) and dyspareunia (25-50\% of women), thus endometriosis represents one of the major causes of morbidity in premenopausal women. Endometriosis of the urinary bladder may result in dysuria or hematuria. The main symptoms of bowel endometriosis are dyschezia and rectorrhagia ${ }^{5,6}$.

The most commonly affected organs are the peritoneum, ovaries, fallopian tubes, uterus, vagina, urinary bladder and rectosigmoideum. In contrast, it is uncommon to find endometriosis in sites such as the cicatrice, umbilicus, omentum, lymph nodes, lungs, heart or bones ${ }^{7,8}$.

The definite cause of endometriosis remains unclear. Many theories have been proposed to explain the pathogenesis of endometriosis and to date they all remain inconclusive (implantation, regurgitation, metaplastic, iatrogenic, immunologic, molecular-genetic theory, theory of tissue injury, vascular and lymphatic dissemination, and stem cell theory) (ref. ${ }^{9-13}$ ).

While considered a benign disorder, endometriosis shares a number of similarities with malignant diseases, such as an abnormal morphology, deregulated cell growth, cellular invasion, neoangiogenesis, DNA aneuploidy, loss of heterozygosity, recurrence, migration along the nerve bundles, metastatic spread to lymph nodes and distant organs ${ }^{14}$. 
Malignant transformation of endometriosis is rare and affects $1 \%$ of lesions with the ovary being the primary site in $79 \%$ (ref. $^{15-16}$ ).

Lymphatic spread of endometriosis to lymph nodes has been documented as a frequent event (25-42\%) in bowel, rectosigmoid and rectovaginal endometriosis ${ }^{17-19}$.

Lymph node involvement by endometriosis consists of isolated endometriotic-like cells (IELC) located in the subcapsular sinus of lymph nodes, representing an early stage of involvement, and frank metastatic endometriotic lesions ${ }^{18}$.

An unusual case of pelvic lymph node endometriosis with an aberrant immunophenotype mimicking metastasis of adenocarcinoma is described as follows:

\section{CASE REPORT}

A 37-year-old woman, smoker, G2P2, with no history or symptoms of endometriosis was referred to our department for invasive cervical adenocarcinoma stage FIGO IA2. Histological examination showed endocervical adenocarcinoma of the usual type, well differentiated, with stromal invasion of $3.5 \mathrm{~mm}$, horizontal spread of $4 \mathrm{~mm}$ and presence of strong nuclear and cytoplasmic expression of $\mathrm{p} 16$; the information about vascular invasion was not included in the pathology report. Cervical cytology specimens were repeatedly classified as atypical squamous cells of undetermined significance (ASCUS). The diagnosis as well as the initial LEEP conization of the cervix with a positive margin were performed at another hospital. The ultrasonographic examination showed normal pelvic area (without free fluid or adhesions). The only positive finding was $36 \times 32 \mathrm{~mm}$ left ovarian cystic lesion, the so called endometrioma. Consequently, the patient underwent a total laparoscopic hysterectomy with pelvic lymphadenectomy at this hospital. Intraoperatively, the diagnosis of deep infiltrating endometriosis of parametrial ligament and vesicouterine pouch, endometrioma of the left ovary and Allen Master's syndrome was suspected (Fig. 1). The histological examination then confirmed the diagnosis.

For classification of endometriosis the revised American Society for Reproductive Medicine (rASRM) score (stage IV) and ENZIAN classification (B1) were used.

\section{Histopathology}

At the department of pathology 6 separate materials were evaluated: left fallopian tube (1), right fallopian tube (2), left ovary (3), uterus including parametrial ligaments (4), right pelvic lymph nodes (5), left pelvic lymph nodes (6). The Materials were extensively investigated using 102 tissue sections stained with hematoxylin-eosin. The uterine cervix was completely processed; no residual epithelial dysplasia or invasive neoplasia was found.
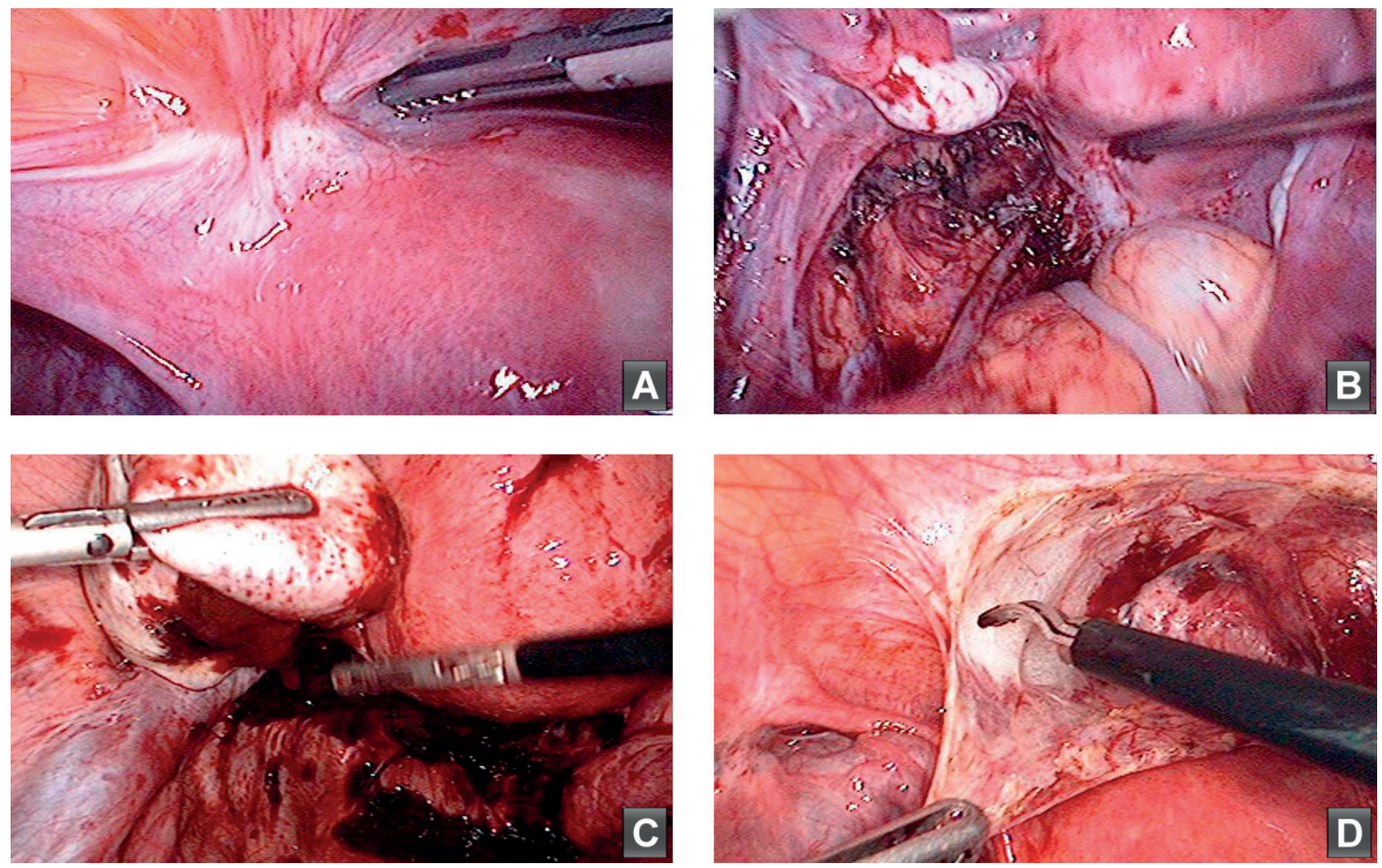

Fig. 1. Intraoperative laparoscopic images of endometriosis. Plica vesicouterina with white endometriosis lesion (A).

Fossa ovarica - uretherolysis, to free the urether from external pressure of adhesions (B).

Endometrioma ovarii - chocolate cyst with haemorrhagic content (C).

Plica vesicouterina with white endometriosis lesion - deperitonealisation (D). 
Endometriosis of the left ovary, uterine cervix, parametrial ligaments and vesicouterine pouch represented an incidental histological finding.

Particularly interesting in relation to the clinical context of cervical adenocarcinoma, was the finding of a solitary gland with cystic dilatation in one of the total of 36 regional pelvic lymph nodes. To determine the nature of the lesion a wide range of immunohistochemical antibodies was used. The gland stained positively with cytokeratin AE1/AE3 (AE1/AE3), cytokeratin 7 (CK7) and negatively with cytokeratin 20 (CK20), p16 (unlike cervical adenocarcinoma), p53, carcinoembryonic antigen (CEA), vimentin, oestrogen and progesterone receptors (ER, PR). Staining with the antibody against CD10 revealed a narrow rim of subjacent stromal cells which coexpressed vimentin, ER and PR (Fig. 2a-c).

For comparative reasons, we found immunoprofile of parametrial ligament endometriosis with the following results - both stromal and glandular immunonegativity for p16, p53, CEA and CK20, only stromal immunopositivity for CD10, only glandular immunopositivity for AE1/ AE3 and CK7, stromal and glandular immunopositivity for vimentin, ER and PR (Fig. 2d,e).

A final diagnosis of lymph node endometriosis with an aberrant immunophenotype was made.

\section{DISCUSSION}

This case report represents a challenging differential diagnosis of glandular lesions in the pelvic lymph nodes.
It was necessary to differentiate between metastasis of cervical and colorectal adenocarcinoma, lymph node endometriosis and benign glandular inclusions of müllerian type. Histologically we found a solitary gland with cystic dilatation lined by a single layer or pseudostratified columnar epithelium with mild nuclear atypia (reactive) and low mitotic rate (without atypical mitotic figures) (Fig. $2 \mathrm{f})$. The diagnosis of malignancy was excluded through a lack of significant cellular atypia and mitotic activity, interfollicular location of the gland and an absence of a desmoplastic stromal reaction.

Müllerian glandular inclusions are found in approximately $5 \%$ of pelvic lymph node dissections performed for staging of malignant neoplasms such as ovarian or cervical carcinoma, or other gynaecological surgeries. These are almost always incidental microscopic findings ${ }^{20-22}$. They are most commonly lined by a single layer of cuboidal to columnar tubal-type epithelium (endosalpingiosis), but sometimes by benign endometrioid epithelium ${ }^{23}$, mucinous epithelium of endocervical ${ }^{24-25}$ or goblet-cell type $^{26}$, or metaplastic squamous epithelium ${ }^{27}$; an admixture of different types of müllerian glands within a lymph node is designated as müllerianosis. Endosalpingiosis is sometimes associated with endometriosis ${ }^{26}$. Therefore the distinction between endometriosis and müllerian glandular inclusions was based on the presence of endometrial stroma.

Using immunohistochemistry, a complete loss of oestrogen and progesterone receptor expression in the endometriotic gland in the pelvic lymph node was identified. From a pathological point of view, this type of aberrant
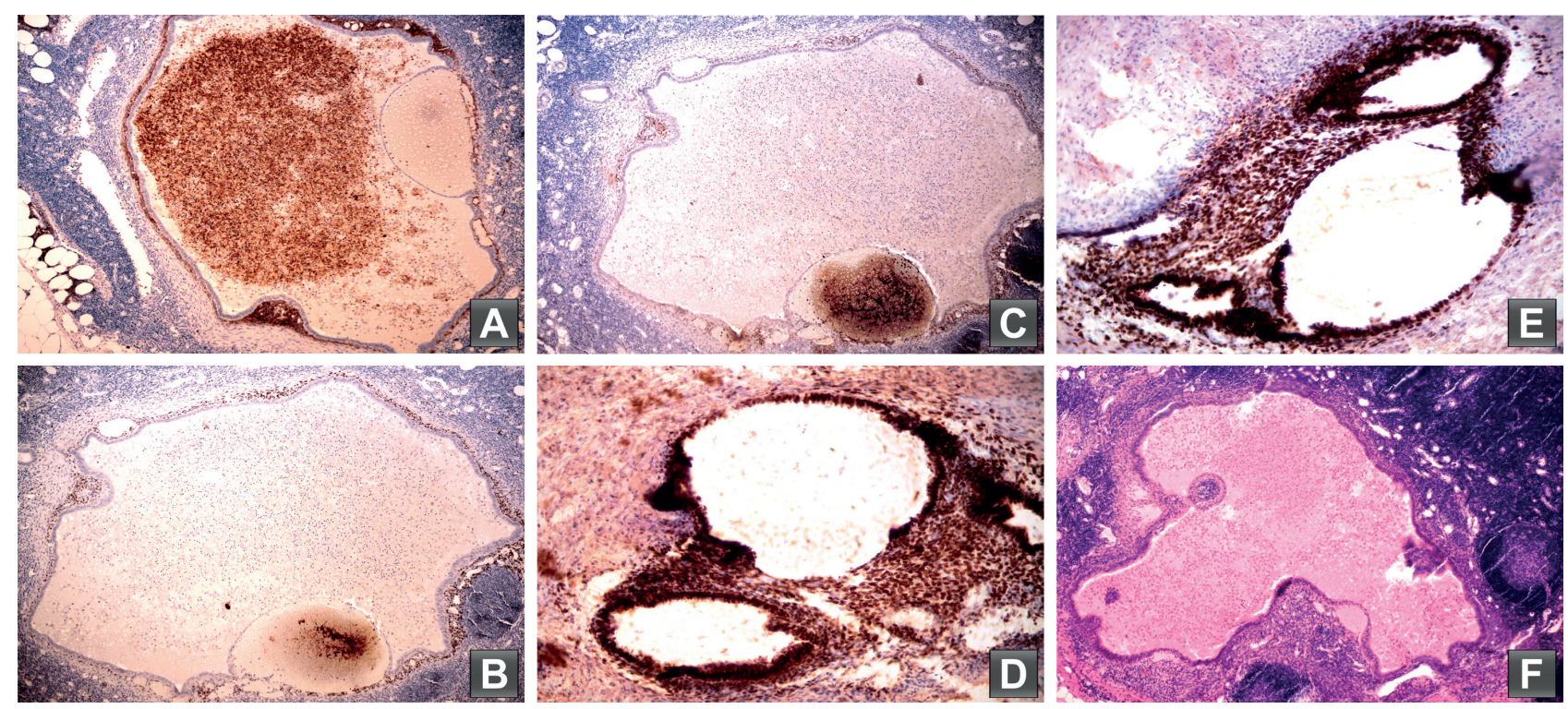

Fig. 2. Immunohistochemical expression of CD10 in lymph node endometriosis, immunohistochemical expression of oestrogen and progesterone receptors in lymph node and parametrial ligament endometriosis and lymph node endometriosis.

CD10 stains a few endometrial stromal cells adjacent to the gland, confirming the diagnosis of lymph node endometriosis (A) (immunohistochemistry, original magnification $\times 100$ ).

In lymph node endometriosis oestrogen (B) and progesterone receptors (C) stain only endometrial stromal cells, endometrial gland remains negative (immunohistochemistry, original magnification $\times 100$ ).

In parametrial ligament endometriosis oestrogen (D) and progesterone receptors (E) stain both endometrial stromal cells and endometrial gland (immunohistochemistry, original magnification $\times 100$ ).

A solitary gland with cystic dilatation lined by a single layer or pseudostratified columnar epithelium with mild reactive atypia without an apparent cuff of stromal endometrial cells (F) (hematoxylin-eosin staining, original magnification x100). 
immunophenotype is very unusual. This phenomenon is not fully understood but possible causes could be metaplastic processes or transformation in atypical endometriosis.

The result of immunohistochemistry may be affected by different methodological approaches. Mouse monoclonal antibodies, which generally show a higher specificity when compared with polyclonal antibodies, were used in this case. Maximum effort was applied to prevent the well known technical and interpretative pitfalls of this methodological approach. All immunohistochemical analyses, including antigen retrieval, were performed in the same laboratory specialized for immunohistochemistry while adhering to standardized protocols. To avoid nonspecific background staining, the system of positive and negative controls was used. For confirmation of this unusual finding, immunohistochemistry was repeatedly performed.

Similar to this study, in 1994 Brandau and co-workers described a case report of an endometriosis in retroperitoneally situated lymph nodes in a 29 -year-old patient. The patient had a cervical carcinoma stage FIGO Ib and was treated surgically by radical hysterectomy after Wertheim-Meigs-Okabayashi with pelvic lymphadenectomy. Coincidentally endometriosis was found in two lymph nodes of the retroperitoneum. The patient had no history or clinical symptoms of endometriosis. Endometriosis could not be found in any other parts of the abdomen. Additionally, an aberrant immunophenotype of the endometriotic gland in a lymph node with only progesterone receptor expression and loss of oestrogen receptor expression was found ${ }^{28}$.

Glandular lesions in the lymph nodes was first described by Ries in 1897 in patients who underwent a hysterectomy and pelvic lymphadenectomy for cervical cancer $^{29}$.

Recently, it has been observed that regional lymph node involvement by endometriosis is a common phenomenon in women with coincidentally found bowel endometriosis $^{30}$.

Endometriotic spread to the lymphatic system is poorly understood and little is known about the molecular events and changes in gene expressions associated with this process. In 2011, Tempfer and colleagues analyzed regional lymph node-spread into pelvic sentinel lymph node (PSLN) in a prospective study of 23 patients with ovarian and/or peritoneal endometriosis. They found endometriotic lesions in $11 \%$ and the presence of IELC in $80 \%$ of PSLN ( ref. $^{31}$ ).

The study by Bürkle and associates, published in 2013, indicates that the spread of endometriosis to PSLN is accompanied by different expressions of several genes, including EPCAM, CDH1 (E-cadherin), CXCR4 and CD44. Using quantitative real-time PCR and immunohistochemistry they analyzed the expression levels of a panel of 28 genes previously described to be associated with endometriosis in a series of samples of primary endometriotic lesions, IELC-negative PSLN and IELC-positive PSLN. Expression of a set of genes (CXCR4, CD68, MKI67 and CD44) was found to be higher in IELC- posi- tive PSLN than in IELC- negative PSLN, while lowest in endometriotic lesions, indicating up-regulation in IELC. In contrast, EPCAM and E-cadherin, which were strongly expressed in endometriotic lesions, were not found to be expressed in IELC- positive PSLN. Study showed that prerequisites for the transition appear to be the up-regulation of the chemokine receptor CXCR4 and CD44 isoforms (including CD44v6 isoform), and the loss of expression of epithelial markers such as E-cadherin and EPCAM. The next step, establishment of an endometriotic lesion with the lymph node, appears to be accompanied by reexpression of epithelial markers such as cytokeratins, E-cadherin and EPCAM (ref. ${ }^{32}$ ). These data are consistent with results published by Ruiz et al. who also found significantly higher CXCR4 expression in endometriotic lesions compared to normal endometrium ${ }^{33}$.

In $2012 \mathrm{Ji}$ published that macrophages act as a direct structure contributor to lymphatic endothelial walls or secrete a vascular endothelial growth factor (VEGF) to initiate lymphangiogenesis in inflamed or tumor tissues ${ }^{34}$. This finding, together with the fact that endometriosis is an inflammatory disorder with an increased concentration of macrophages, indicates the possible role of macrophages in the process of lymph node involvement by endometriosis.

To our knowledge, the scientific literature does not describe endometriosis with an aberrant immunophenotype with complete loss of oestrogen and progesterone receptor expression.

Immunohistochemical hormonal receptor expression in eutopic and ectopic endometrium was analyzed by Jones and co-workers in a series of 30 women with endometriosis. They found an increased oestrogen receptor expression in ectopic endometrium from the proliferative to the late secretory phase. Compared with the eutopic endometrium, the expression in both epithelium and stroma of ectopic endometrium was significantly higher throughout the cycle. In contrast, stromal progesterone receptor expression tended to be reduced in ectopic endometrium compared with eutopic tissue, while epithelial progesterone receptor expression was increased in ectopic endometrium only in the late secretory phase ${ }^{35}$. Despite the variable intensity of hormonal receptor expression, all cases showed typical immunophenotype with immunopositivity for both oestrogen and progesterone receptors. In searching the literature, no publication describing heterogeneous expression of estrogen and progesterone receptors in eutopic and ectopic endometrium was found.

Mechsner et al. retrospectively analyzed the frequency of endometriotic lesions and disseminated endometrioticlike cells in a series of 108 coincidentally resected lymph nodes of 24 patients with endometriosis. Endometriotic lesions were detected in 8 out of 24 patients, while disseminated endometriotic-like cells were found in 17 out of the 24 patients. The study showed typical immunophenotype in all cases (endometriotic lesions stained positively with ER, PR, CD10 and cytokeratin, endometriotic-like cells stained positively with ER and PR) ( $r e f .{ }^{36}$ ).

Currently, little is known about the mechanism of 
recurrence of endometriosis and its management is not efficacious. After surgical and/or medical therapy, endometriosis has a high recurrence rate which increases with the length of follow-up. To delay or eliminate recurrence is the main goal in the control of this disease ${ }^{30}$. Only a limited number of studies investigated the clinical significance of lymph node involvement by endometriosis with somewhat conflicting results. The most recent study by Rossini and colleagues from 2016 analyzed correlation between the severity of endometriosis and lymph node involvement. The study included 140 cases of colorectal surgery for intestinal endometriosis; histopathological examination revealed the involvement in $50 \%$ of the cases. They showed no statistically significant correlation between the positivity of lymph nodes and the rate of intestinal stenosis or the histopathological specimen infiltration rate and depth and the intestinal recurrence rate. Based on these results, they hypothesized that lymph node involvement in intestinal resection specimens does not modify the natural history of the disease ${ }^{37}$. Conversely, in the study by Gong and Tempfer, endometriotic cells in regional lymph nodes were described as a potential target of hormonal stimulation in the postoperative period and they could be a major source of disease recurrence ${ }^{30}$.

In 2011 Namkung at al. reported that rectal endometriosis has the ability to invade adjacent tissue as true malignant tumors and therefore, lymph node involvement should be considered in rectal endometriosis ${ }^{38}$.

\section{CONCLUSION}

The coincidence of a cervical adenocarcinoma and endometriosis in pelvic lymph nodes may represent a differential diagnostic problem. Confirmation of the correct diagnosis requires the use of a wide range of immunohistochemical antibodies. In the case of an aberrant immunophenotype with loss of oestrogen and progesterone receptor expression of the endometriotic gland, the finding of CD10-positive endometrial stromal cells and benign morphology of the glandular lesion is essential for proper diagnosis. To our knowledge, this type of aberrant immunophenotype of the endometriotic gland has never been presented in any scientific literature. This finding plays a significant role from a pathology standpoint and perhaps, even more importantly, from the clinical standpoint. The asymptomatic patient with the correct diagnosis of lymph node endometriosis did not undergo excessive treatment for false positive diagnosis of metastatic cervical adenocarcinoma.

Currently, little is known about the mechanism of lymph node spread of endometriosis. The question remains whether lymph node endometriosis is a significant prognostic factor and will affect the therapeutic approach to this disorder in the future. Thus, the presence of lymph node involvement with endometriotic cells may perhaps point toward a systemic aspect of this disease and the resection of regional lymph nodes could decrease the recurrence rate of endometriosis.

\section{ABBREVIATIONS}

AE1/AE3, cytokeratin AE1/AE3; ASCUS, Atypical squamous cells of undetermined significance; CDH1, Cadherin 1; CD10, Cluster of differentiation 10; CD44, Cluster of differentiation 44; CD68, Cluster of differentiation 68; CEA, Carcinoembryonic antigen; CK7, Cytokeratin 7; CK20, Cytokeratin 20; CXCR4, C-X-C chemokine receptor type 4; DNA, Deoxyribonucleic acid; EPCAM, Epithelial cell adhesion molecule; ER, Oestrogen receptors; FIGO, International federation of gynecology and obstetrics; G2P2, Gravida 0 para 0; IELC, Isolated endometriotic-like cells; IELC- negative PSLN, Isolated endometriotic-like cells negative pelvic sentinel lymph node; IELC- positive PSLN, Isolated endometriotic-like cells positive pelvic sentinel lymph node; LEEP, Loop electrosurgical excision procedure; PCR, Polymerase chain reaction; PR, Progesterone receptors; PSLN, Pelvic sentinel lymph node; $\mathrm{p} 16$, protein 16; p53, protein 53; rASRM, revised American society for reproductive medicine score; VEGF, Vascular endothelial growth factor.

Author contributions: DV: project development, manuscript writing, description of the laparoscopic images; JL: manuscript writing, description of the histopathology findings; $\mathrm{RCH}$ : description of the laparoscopic images, critically revising the article; JT, MK: literature search; LU: linguistic review, manuscript editing.

Conflict of interest statement: The authors state that there are no conflicts of interest regarding the publication of this article.

\section{REFERENCES}

1. Munksgaard PS, Blaakaer J. The association between endometriosis and gynecological cancers and breast cancer: a review of epidemiological data. Gynecol Oncol 2011;123(1):157-63.

2. Sayasneh A, Tsivos D, Crawford R. Endometriosis and ovarian cancer: a systematic review. ISRN Obstet Gynecol 2011;2011:140310.

3. Somigliana E, Vigano P, Parazzini F, Stoppelli S, Giambattista E, Vercellini P. Association between endometriosis and cancer: a comprehensive review and a critical analysis of clinical and epidemiological evidence. Gynecol Oncol 2006;101(2):331-41.

4. Nezhat F, Datta MS, Hanson V, Pejovic T, Nezhat C, Nezhat C. The relationship of endometriosis and ovarian malignancy: a review. Fertil Steril 2008;90(5):1559-70

5. Treloar SA, O'Connor DT, O'Connor VM, Martin NG. Genetic influences on endometriosis in an Australian twin sample. Fertil Steril 1999;71(4):701-10.

6. Giudice LC, Kao LC. Endometriosis. Lancet 2004;364(9447):1789-99.

7. Giudice LC. Clinical practice. Endometriosis. N Engl J Med 2010; 362(25):2389-98.

8. De Ceglie A, Bilardi C, Blanchi S, Picasso M, Di Muzio M, Trimarchi A, Conio M. Acute small bowel obstruction caused by endometriosis: a case report and review of the literature. World J Gastroenterol 2008; 14(21):3430-4.

9. Gargett CE, Schwab KE, Zillwood RM, Nguyen HP, Wu D. Isolation and culture of epithelial progenitors and mesenchymal stem cells from human endometrium. Biol Reprod 2009;80(6):1136-45.

10. Maruyama T, Yoshimura Y. Stem cell theory for the pathogenesis of endometriosis. Front Biosci (Elite Ed) 2012;4:2854-63.

11. Iwanoff $S$. Adenofibromyoma cysticum sarcomatoides carcinomatosum. Monatschr Geburtshilfe Gynakol 1898;7:295. 
12. Sampson J. Peritoneal endometriosis due to the menstrual dissemination of endometrial tissue into the peritoneal cavity. Am J Obstet Gynecol 1927;14:442-69.

13. Javert CT. Pathogenesis of endometriosis based on endometria homeoplasia, direct extension, exfoliation and implantation, lymphatic and hematogenous metastasis, including five case reports of endometrial tissue in pelvic lymph nodes. Cancer 1949;2(3):399-410.

14. Thomas EJ, Campbell IG. Molecular genetic defects in endometriosis. Gynecol Obstet Incest 2000;50 Suppl 1:44-50.

15. Higashiura $\mathrm{Y}$, Kajihara H, Shigetomi H, Kobayashi H. Identification of multiple pathways involved in the malignant transformation of endometriosis (Review). Oncol Lett 2012;4(1):3-9.

16. Heaps JM, Nieberg RK, Berek JS. Malignant neoplasms arising in endometriosis. Obstet Gynecol 1990;75(6):1023-8.

17. Insabato L, Pettinato $G$. Endometriosis of the bowel with lymph node involvement. A report of three cases and review of the literature. Pathol Res Pract 1996;192(9):957-61.

18. Mechsner S, Weichbrodt M, Riedlinger WF, Kaufmann AM, Schneide A, Köhler C. Immunohistochemical evaluation of endometriotic lesions and disseminated endometriosis-like cells in incidental lymph nodes of patients with endometriosis. Fertil Steril 2010;94(2):457-63.

19. Noël JC, Chapron C, Fayt I, Anaf V. Lymph node involvement and lymphovascular invasion in deep infiltrating rectosigmoid endometriosis. Fertil Steril 2008;89(5):1069-72.

20. Karp LA, Czernobilsky B. Glandular inclusions in pelvic and abdominal para-aortic lymph nodes. A study of autopsy and surgical material in males and females. Am J Clin Pathol 1969;52(2):212-8.

21. Kheir SM, Mann WJ, Wilkerson JA. Glandular inclusions in lymph nodes. The problem of extensive involvement and relationship to salpingitis. Am J Surg Pathol 1981;5(4):353-9.

22. Horn LC, Bilek K. Frequency and histogenesis of pelvic retroperitone al lymph node inclusions of the female genital tract. An immunohistochemical study of 34 cases. Pathol Res Pract 1995;191(10):991-6.

23. Lauchlan SC. The secondary müllerian system. Obstet Gynecol Surv 1972;27(3):133-46

24. Baird DB, Reddick RL. Extraovarian mucinous metaplasia in a patient with bilateral mucinous borderline ovarian tumors: a case report. Int J Gynecol Pathol 1991;10(1):96-103.

25. Sinkre $P$, Hoang MP, Albores-Saavedra J. Mullerianosis of inguina lymph nodes: report of a case. Int J Gynecol Pathol 2002;21(1):60-4
26. Tazelaar HD, Vareska G. Benign glandular inclusions. Hum Pathol 1986;17(1):100-1.

27. Mills SE. Decidua and squamous metaplasia in abdominopelvic lymph nodes. Int J Gynecol Pathol 1983;2(2):209-15.

28. Regidor-Brandau PA, Pfaffenbach B, Metz KA, Schindler AE. [Endometriosis in retroperitoneal lymph nodes]. Geburtshilfe Frauenheilkd 1994;54(6):372-4.

29. Ries E. Eine neue Operationsmethode des Uteruskarzinoms Z Geburtsh Genak 1897;518-532.

30. Gong Y, Tempfer CB. Regional lymphatic spread in women with pelvic endometriosis. Med Hypothese 2011;76(4):560-3.

31. Tempfer CB, Wenzl R, Horvat R, Grimm C, Polterauer S, Buerkle B, Reinthaller A, Huber JC. Lymphatic spread of endometriosis to pelvic sentinel lymph nodes: a prospective clinical study. Fertil Steril 2011;96(3):692-6.

32. Bürkle B, Notscheid NK, Scheich J, Hefler L, Tempfer CB, Horvat R, Rezniczek GA. Spread of endometriosis to pelvic sentinel lymph nodes: gene expression analysis. Eur J Obstet Gynecol Reprod Biol 2013;169(2):370-5.

33. Ruiz A, Salvo VA, Ruiz LA, Báez P, García M, Flores I. Basal and steroid hormone-regulated expression of CXCR4 in human endometrium and endometriosis. Reprod Sci 2010;17(10):894-903.

34. Ji RC. Macrophages are important mediators of either tumor- or inflammation-induced lymphangiogenesis. Cell Mol Life Sci 2012;69(6):897-914.

35. Jones RK, Bulmer JN, Searle RF. Immunohistochemical characterization of proliferation, oestrogen receptor and progesterone receptor expression in endometriosis: comparison of eutopic and ectopic endometrium with normal cycling endometrium. Hum Reprod 1995;10(12):3272-9.

36. Mechsner S, Weichbrodt M, Riedlinger WF, Kaufmann AM, Schneider A, Köhler C. Immunohistochemical evaluation of endometriotic lesions and disseminated endometriosis-like cells in incidental lymph nodes of patients with endometriosis. Fertil Steril 2010;94(2):457-63.

37. Rossini R, Monsellato D, Bertolaccini L, Pesci A, Zamboni G, Ceccaroni M, Ruffo G. Lymph Node Involvement in Deep Infiltrating Intestinal Endometriosis: Does It Really Mean Anything? J Minim Invasive Gynecol 2016; 23(5):787-92.

38. Namkung J, Kim SJ, Kim JH, Kim J, Hur SY. Rectal endometriosis with invasion into lymph nodes. J Obstet Gynaecol Res 2011;37(8):111721. 